\title{
An Optimization-Based Method for Bounding State Functionals of Nonlinear Stochastic Systems
}

\author{
Mohamadreza Ahmadi, Andreas W. K. Harris, Antonis Papachristodoulou
}

\begin{abstract}
We propose a method for bounding state functionals of a class of nonlinear stochastic differential equations. Given a class of state functionals of a stochastic system, the Feynman-Kac Lemma presents a backward in time partial differential equation that describes the evolution of the state functional. We bound these state functionals based on a method which uses barrier functionals. We show that, under the assumption of polynomial data, the bounds can be obtained by semi-definite programming. The proposed method is then applied to the case study of noise in genetic negative autoregulation to bound a functional of the second moment, which is of specific interest to experimental assays. The bound yielded, using biological parameters, is found to be in good agreement with experimental results in the literature.
\end{abstract}

\section{INTRODUCTION}

The complexity of many dynamical systems in nature and engineering results in models that are described by stochastic differential equations (SDEs). For example, in biochemical interactions, where the occurrence of reactions due to thermal fluctuations is a random process, deterministic models fail to capture the dynamics properly, especially in the case of small species populations [1].

Finding explicit solutions to nonlinear SDEs is a cumbersome task in general. Hence, numerical methods, such as the Euler-Maruyama method [2], are used to approximate solutions. Yet, for significant classes of SDEs, approximating solutions is too computationally demanding, in particular, if there is uncertainty in the parameters or initial conditions. Fortunately, Lyapunov methods can be used to study stability and convergence properties of SDEs without the need to approximate solutions [3], [4].

Rather than studying stability, in many important applications, we are merely interested in evaluating the moments or integral functionals of the moments at particular points in time. It is well known that functionals of the solutions of the stochastic models for asset prices describe the price of an option [5]. For nonlinear SDEs, finding the dynamics of the statistical moments is not trivial, because the dynamics of the lower-order moments depend on the higher-order moments leading to the moment closure problem. This problem has been studied extensively in the context of biological

The authors are with the Department of Engineering Science, University of Oxford, Oxford, OX1 3PJ, UK e-mail: (\{mohamadreza.ahmadi, andreas.harris, antonis $\} @$ eng.ox.ac.uk). M. Ahmadi is supported by the Clarendon Scholarship and the Sloane-Robinson Scholarship. A. Harris was supported by the Engineering and Physical Sciences Research Council (EPSRC) Doctoral Training Centre in Systems Biology, University of Oxford. A. Papachristodoulou was supported in part by the EPSRC projects EPJ012041/1, EP/I031944/1, EP/M002454/1, and EP/J010537/1. applications [6], [7] and, in particular, biochemical reaction networks [8], [9].

In this paper, we propose a method for bounding state functionals of a class of nonlinear SDEs. The method is based on a generalized version of the Feynman-Kac Lemma, which describes the backward dynamics of a cost functional of the moments of an SDE. The main tool is the barrier functional [10], which is a generalization of barrier certificates [11], [12], [13] to infinite-dimensional systems. We demonstrate that if the barrier functional satisfies two inequalities along the solutions of the backward dynamics, then we can infer bounds on the cost functional of the moments. We further show that, under the assumption of polynomial data, the proposed method can be cast as semidefinite programs (SDPs). In this regard, we generalize our earlier results in [14] and in [15] to integral inequalities with time dependence. Furthermore, we formulate an Sprocedure-like theorem for integral inequalities.

The proposed method is applied to the case study of negative autoregulation. This gene circuit motif implements the simplest form of biological negative feedback. As understanding of biological systems with feedback increases [16], [17], there is an ever greater need for the application of theoretical tools to the fields of systems and synthetic biology. Negative autoregulation is well studied and ubiquitous in nature [18], [19], [20], [21]. Though it has been shown that negative autoregulation experimentally reduces rise time [19], measuring the dynamics of such systems in an experimental context continues to be challenging. On the other hand, noise data, at both culture and single cell level, is a common and reliable tool [18], [20], [21]. This motivates the need for accurate theoretical tools to calculate and predict the concentration distributions of studied proteins across a populations of cells.

The rest of this paper is as follows. In Section II, we briefly review some background definitions and theorems. In Section III, we describe the proposed method for bounding state functionals of SDEs using barrier functionals. We then propose a computational method based on SDPs to find these bounds. Section IV considers the application of the proposed method to find bounds on a functional of the second moment of a model of negative autoregulation. Finally, Section V concludes the paper.

\section{Notation:}

The $n$-dimensional Euclidean space is denoted by $\mathbb{R}^{n}$ and the set of non-negative reals by $\mathbb{R}_{\geq 0}$. The $n$-dimensional set of positive integers is denoted by $\overline{\mathbb{N}}^{n}$, and the $n$-dimensional space of non-negative integers is denoted by $\mathbb{N}_{>0}^{n}$. The 
notation $M^{\prime}$ denotes the transpose of matrix $M$ and $\operatorname{Tr}\{M\}$ is the trace of the square matrix $M$. A domain $\Omega$ is an open subset of $\mathbb{R}^{n}$ with $\mathcal{C}^{1}$ boundary $\partial \Omega$. The ring of polynomials on real variable $x \in \mathbb{R}^{n}$ and $y \in \mathbb{R}^{m}$ is denoted $\mathcal{R}[x, y]$. The space of $k$-times continuous differentiable functions defined on $\Omega$ is denoted by $\mathcal{C}^{k}(\Omega)$ and the space of $\mathcal{C}^{k}(\Omega)$ functions mapping to a set $\Gamma$ is denoted $\mathcal{C}^{k}(\Omega ; \Gamma)$. For a multivariable function $f(x, y)$, we use the notation $f(x, \cdot) \in \mathcal{C}^{k}[x]$ to show $k$-times continuous differentiability of $f$ with respect to variable $x$. If $p \in \mathcal{C}^{1}(\Omega)$, then $\partial_{x} p$ denotes the derivative of $p$ with respect to variable $x \in \Omega$. In addition, we adopt Schwartz's multi-index notation. For $u \in \mathcal{C}^{k}\left(\Omega ; \mathbb{R}^{n}\right), \alpha \in$ $\mathbb{N}_{0}^{n}$, define

$$
D^{\alpha} u:=\left(u_{1}, \partial_{x} u_{1}, \ldots, \partial_{x}^{\alpha_{1}} u_{1}, \ldots, u_{n}, \partial_{x} u_{n}, \ldots, \partial_{x}^{\alpha_{n}} u_{n}\right) .
$$

For functions $f \in \mathcal{C}^{1}(\Omega)$ and $g \in \mathcal{C}^{2}(\Omega), \nabla f$ denotes the gradient vector and $\nabla^{2} g$ denotes the Hessian matrix. For a random variable $X, E\{X\}$ denotes its expected value.

\section{PRELIMINARIES}

Let $T>0$ and let $\left(\Gamma, \mathcal{J},\left\{\mathcal{J}_{s}\right\}_{s>0}, \mathrm{P}\right)$ be a complete and right-continuous filtered probability space, where $\Gamma$ is a sample space, $\left\{\mathcal{J}_{s}\right\}_{s \geq 0}$ with $\mathcal{J}_{s} \subseteq \mathcal{J}$ for each $s$ is a filteration of the $\sigma$-algebra $\mathcal{J}$, and $\mathrm{P}$ is the probability measure function. Consider the following SDE

$$
\left\{\begin{array}{l}
\mathrm{d} X(s)=f(s, X(s)) \mathrm{d} s+g(s, X(s)) \mathrm{d} W(s), \quad s \in[t, T], \\
X(t)=x,
\end{array}\right.
$$

where $x$ is a $\mathcal{J}_{t}$-measurable random variable, $X(s) \in \Omega \subset$ $\mathbb{R}^{d}$ denotes the states and $W(s) \in \mathbb{R}^{m}$ is an $m$-dimensional standard $\left\{\mathcal{J}_{s}\right\}_{s \geq 0}$-Wiener process starting at $t$ (i.e., $W(t)=$ $0)$. Moreover, consider the following backward in time PDE

$$
\begin{aligned}
-\partial_{t} u(t, x)= & \frac{1}{2} \operatorname{Tr}\left\{g(t, x) g^{\prime}(t, x) \nabla^{2} u(t, x)\right\} \\
& +f^{\prime}(t, x) \nabla u(t, x)+c(t, x) u(t, x) \\
& +h(t, x), \quad(t, x) \in[0, T) \times \Omega, \\
u(T, x)= & q(x) .
\end{aligned}
$$

Assumption I: The maps $f:[0, T] \times \Omega \rightarrow \mathbb{R}^{d}$ and $g:$ $[0, T] \times \Omega \rightarrow \mathbb{R}^{d \times m}, c, h:[0, T] \times \Omega \rightarrow \mathbb{R}$, and $q: \Omega \rightarrow \mathbb{R}$ are uniformly continuous, $c$ is bounded, and there exists a constant $L>0$ such that for $\phi=f, g, c, h$,

$$
\begin{cases}|\phi(t, x)-\phi(t, \hat{x})| \leq L|x-\hat{x}|, & \forall t \in[0, T], x, \hat{x} \in \Omega \\ |\phi(t, 0)| \leq L, & \forall t \in[0, T] .\end{cases}
$$

Remark 1: If Assumption I holds, there exists a unique

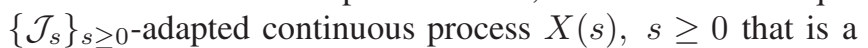
unique strong solution to SDE (1) (see Definition 6.2 in [22]).

Define

$$
\tau:=\inf \{s \in[t, T] \mid X(s) \notin \Omega\} .
$$

We recall the following result (Theorem 4.2, p. 374 in [22] and Theorem 7.6, p. 366 in [23]), which is a generalized version of the Feynman-Kac Lemma.
Theorem 1: Consider (2) with boundary conditions $\left.u\right|_{\partial \Omega}=\psi(t, x)$ and SDE (1). Let Assumption I hold. Let

$$
\Psi(t, x)= \begin{cases}q(x), & (t, x) \in[0, T] \times \Omega, \\ \psi(t, x), & (t, x) \in[0, T] \times \partial \Omega,\end{cases}
$$

be a continuous function on $([0, T] \times \Omega) \cup([0, T] \times \partial \Omega)$. Then, (2) with boundary conditions $\left.u\right|_{\partial \Omega}=\psi(t, x)$ admits a unique viscosity solution given by

$$
\begin{array}{r}
u(t, x)=E\left\{\int_{t}^{\tau} h(s, X(s)) e^{-\int_{t}^{s} c(r, X(r)) \mathrm{d} r} \mathrm{~d} s\right. \\
\left.+\Psi(\tau, X(\tau)) e^{-\int_{t}^{\tau} c(r, X(r)) \mathrm{d} r} \mid X(t)=x\right\}, \\
(t, x) \in[0, T] \times \Omega,
\end{array}
$$

where $X$ is the unique strong solution of $\operatorname{SDE}$ (1). In addition, if (1) admits a classical solution, then (6) is a classical solution to (2).

Remark 2: Consider equation (2) with $c, h=0$. This is equivalent to the backward Kolmogorov equation [24, Chapter 4], for which we have

$$
u(t, x)=E\{q(X(T)) \mid X(t)=x\},
$$

where $X$ satsfies (1).

The function $u(t, x)$ in (6) specifies the value of the functional

$$
E\left\{\int_{t}^{\tau} h(s, X(s)) e^{-\int_{t}^{s} c(r, X(r)) \mathrm{d} r} \mathrm{~d} s+\Psi(\tau, X(\tau))\right\},
$$

for a solution of SDE (1) starting at time $t$ with initial condition $x$. Indeed, Theorem 1 relates the solutions of the SDE (1) to the solution of the backward PDE (2) through functional (6). The functional given in (6) encompasses a rich class of state functionals of SDE (1). For instance, for $c=0, h(s, X(s))=X^{3}(s)$ and $q(X(T))=X^{3}(T)$,

$$
u(0, x)=E\left\{\int_{0}^{T} X^{3}(s) \mathrm{d} s+X^{3}(T) \mid X(0)=x\right\},
$$

represents the finite-time cost functional with a terminal value of the third moment of the solutions to SDE (1).

\section{Main Results}

In this section, we propose a method for bounding state functionals of SDEs based on barrier functionals, which reduces to solving an optimization problem. Moreover, we show that, in the case of polynomial data, the optimization problem can be cast as an SDP.

\section{A. Bounding State Functionals of SDEs using Barrier Func- tionals}

Let $\mathcal{U} \subseteq \mathcal{C}^{1}[t] \cap \mathcal{C}^{2}[x]$. We define the Barrier Functional

$$
B(t, u)=\mathscr{B}(t) u,
$$

where $\mathscr{B}(t): \mathcal{U} \rightarrow \mathbb{R}$ is a possibly nonlinear operator.

Finding bounds on the moments of the nonlinear SDE (1) is not straightforward due to the moment closure problem. In addition, when (1) is nonlinear, solving the backward 
PDE (2) in general is cumbersome. In the following, we propose a method for bounding the solutions of PDE (2) and hence state functionals of (1).

We begin by showing that if the barrier functional (7) satisfies two inequalities along the solutions of the backward system (2), we can ensure that the solutions of (2) avoid an undesirable set.

Theorem 2: Consider the backward PDE (2). Given a set of terminal conditions

$$
\mathcal{U}_{T}=\{u \in \mathcal{U} \mid u(T, x)=q(x)\},
$$

an undesirable set $\mathcal{Y}_{u}$ such that $\mathcal{U}_{T} \cap \mathcal{Y}_{u}=\emptyset$, and $t_{0} \in[0, T]$, if there exists a barrier functional $B(t, u(t, x)) \in \mathcal{C}^{1}[t]$ as in (7) such that the following inequalities hold

$$
\begin{gathered}
B\left(t_{0}, u\left(t_{0}, x\right)\right)-B(T, u(T, x))>0 \\
\forall u\left(t_{0}, x\right) \in \mathcal{Y}_{u}, \forall u(T, x) \in \mathcal{U}_{T} \\
\frac{\mathrm{d} B(t, u(t, x))}{\mathrm{d} t} \geq 0, \forall t \in[0, T], \forall u \in \mathcal{U}
\end{gathered}
$$

along the solutions of (2), then the solutions $u(t, x)$ of (2) satisfy $u\left(t_{0}, x\right) \notin \mathcal{Y}_{u}$ for $t_{0} \in[0, T]$.

Proof: We prove the theorem by contradiction. Assume that at time $t_{0} \in[0, T]$, there exists a solution $u(t, x)$ to (2) with $u(T, x) \in \mathcal{U}_{T}$ that satisfies $u\left(t_{0}, x\right) \in \mathcal{Y}_{u}$. Then, from (9a), we have

$$
B\left(t_{0}, u\left(t_{0}, x\right)\right)-B(T, u(T, x))>0 .
$$

On the other hand, inequality (9b) implies that for all $t \in$ $[0, T]$, it holds that $\frac{\mathrm{d} B(t, u(t, x))}{\mathrm{d} t} \geq 0$. Integrating both sides of the latter inequality, from $t$ to $T$, yields

$$
\int_{t}^{T} \frac{\mathrm{d} B(t, u(t, x))}{\mathrm{d} t} \mathrm{~d} t=B(T, u(T, x))-B(t, u(t, x)) \geq 0 .
$$

Since $t, t_{0} \in[0, T]$, this contradicts (10). Therefore, there is no solution to (2) that satisfies $u\left(t_{0}, x\right) \in \mathcal{Y}_{u}$.

In the next Corollary, we show how Theorem 2 can be used for bounding state functionals of SDE (1). We use an appropriate definition for the undesirable set $\mathcal{Y}_{u}$ and a corresponding optimization problem.

Corollary 1: Consider PDE (2) and SDE (1). Let

$$
\mathcal{Y}_{u}=\left\{u \in \mathcal{U} \mid u\left(t_{0}, x\right)>\gamma\right\} \text {. }
$$

If there exists a barrier functional $B(t, u(t, x)) \in \mathcal{C}^{1}[t]$ such that inequalities (9a) and (9b) are satisfied along the solutions of (2), then we have

$$
\begin{aligned}
& E\left\{\int_{t_{0}}^{\tau} h(s, X(s)) e^{-\int_{t_{0}}^{s} c(r, X(r)) \mathrm{d} r} \mathrm{~d} s\right. \\
& \left.+\Psi(\tau, X(\tau)) e^{-\int_{t_{0}}^{\tau} c(r, X(r)) \mathrm{d} r} \mid X\left(t_{0}\right)=x\right\} \leq \gamma .
\end{aligned}
$$

Proof: If there exists a barrier functional $B(t, u(t, x)) \in$ $\mathcal{C}^{1}[t]$ such that inequalities $(9 a)$ and $(9 b)$ are satisfied along the solutions of (2), from Theorem (2), we can infer that $u\left(t_{0}, x\right) \notin \mathcal{Y}_{u}$ with $\mathcal{Y}_{u}$ as described by (11). Thus, $u\left(t_{0}, x\right) \leq$ $\gamma$. From Theorem 1, we have

$$
\begin{array}{r}
u\left(t_{0}, x\right)=E\left\{\int_{t_{0}}^{\tau} h(s, X(s)) e^{-\int_{t_{0}}^{s} c(r, X(r)) \mathrm{d} r} \mathrm{~d} s\right. \\
\left.+\Psi(\tau, X(\tau)) e^{-\int_{t_{0}}^{\tau} c(r, X(r)) \mathrm{d} r} \mid X(t)=x\right\},
\end{array}
$$

Therefore, $u\left(t_{0}, x\right) \leq \gamma$ implies that (12) holds.

In order to find the upper bound on the state functional, i.e., minimum $\gamma$ in (12), we solve the following optimization problem

$$
\begin{gathered}
\min _{B(t, u(t, x))} \gamma \\
\text { subject to (9a) and (9b). }
\end{gathered}
$$

Remark 3: If the domain $\Omega$ is chosen such that for all $s \in[0, T], X(s) \in \Omega$, we can replace $\tau$ with $T$ and $\Psi(t, x)=$ $q(x)$ in Theorem 1 and Corollary 1. Then, we have

$$
\begin{aligned}
u\left(t_{0}, x\right)=E & \left\{\int_{t_{0}}^{T} h(s, X(s)) e^{-\int_{t_{0}}^{s} c(r, X(r)) \mathrm{d} r} \mathrm{~d} s\right. \\
& \left.+q(X(T)) e^{-\int_{t_{0}}^{T} c(r, X(r)) \mathrm{d} r} \mid X(t)=x\right\} .
\end{aligned}
$$

Remark 4: In [25], an SDP-based method for bounding the moments of continuous-time Markov chains, based on the Foster-Lyapunov stability theory (see condition CD2' in [26]), is proposed. Continuous-time Markov chains can be represented by the Chemical Master Equations (CMEs) [27], which are a set of ODEs. When the system is sufficiently large, CMEs can be approximated by a set of SDEs called Chemical Langevin Equations (CLEs) [28]to which the method studied in this paper can be applied to find bounds.

\section{B. Computational Method Based on SDPs}

This section focuses on the computation of barrier functionals satisfying the conditions of Theorem 2 and Corollary 1 using semi-definite programming under some assumptions. In this respect, we reformulate the problem of checking positivity of time-dependent integral inequalities into solving SDPs.

1) Solving Integral Inequalities in 1D: This subsection presents conditions for the verification of time-dependent integral inequalities, defined in a bounded interval. These conditions are obtained by considering a quadratic-like representation of the integrand and differential relations among the dependent variables. As a result, the positivity of the integral is checked via the positivity of a matrix function, describing the quadratic form in the integrand, over the domain of integration. The conditions and the main steps for their derivation are presented below. These steps are all automated and available as a plug-in to SOSTOOLS [29].

Let $\Omega=(a, b) \subset \mathbb{R}$. Consider the following inequality

$$
\begin{aligned}
\mathcal{F}=\int_{a}^{b}\left(D^{\alpha} u\right)^{\prime} F(t, x)\left(D^{\alpha} u\right) \mathrm{d} x & -\left[\left(D^{\alpha-1} u(t, b)\right)^{\prime} F_{1}(t)\left(D^{\alpha-1} u(t, b)\right)\right. \\
& \left.\quad-\left(D^{\alpha-1} u(t, a)\right)^{\prime} F_{0}(t)\left(D^{\alpha-1} u(t, a)\right)\right] \geq 0 .
\end{aligned}
$$


with $F: \mathbb{R}_{\geq 0} \times(a, b) \rightarrow \mathbb{S}^{n_{\alpha}}, n_{\alpha}=\sum_{k=1}^{n} \alpha_{k}$, $F_{i}(t): \mathbb{R}_{\geq 0} \rightarrow \mathbb{S}^{n_{\alpha-1}}, n_{\alpha-1}=\sum_{k=1}^{n}\left(\alpha_{k}-1\right), i=0,1$ and the dependent variable $u$ satisfying

$$
u \in \mathcal{U}_{s}(Q):=\left\{u \mid Q\left[\begin{array}{c}
D^{\alpha-1} u(t, b) \\
D^{\alpha-1} u(t, a)
\end{array}\right]=0\right\},
$$

where $Q \in \mathbb{R}^{n \alpha \times 2 n \alpha}$. In the following, we show how to account for (16) when solving (15). The lemma below establishes a relation between the values at the boundary $u(t, b)$ and $u(t, a)$ and the integrand and is a straightforward application of the Fundamental Theorem of Calculus. It will be used to introduce extra terms in the integral in (15).

Lemma 1: Consider a matrix function $H(t, x) \in \mathcal{C}^{1}[x]$, $H: \mathbb{R}_{\geq 0} \times(a, b) \rightarrow \mathbb{S}^{n_{\alpha-1}}$. We have

$$
\begin{gathered}
\int_{a}^{b} \frac{\mathrm{d}}{\mathrm{d} x}\left[\left(D^{\alpha-1} u\right)^{\prime} H(t, x)\left(D^{\alpha-1} u\right)\right] \mathrm{d} x \\
=\quad \int_{a}^{b}\left(D^{\alpha-1} u\right)^{\prime} \frac{\partial H(t, x)}{\partial x}\left(D^{\alpha-1} u\right) \\
\quad+2\left(D^{\alpha-1} u\right)^{\prime} H(t, x)\left(D^{\alpha} u\right) \mathrm{d} x \\
=\quad\left(D^{\alpha-1} u(t, b)\right)^{\prime} H(t, b)\left(D^{\alpha-1} u(t, b)\right) \\
\quad-\left(D^{\alpha-1} u(t, a)\right)^{\prime} H(t, a)\left(D^{\alpha-1} u(t, a)\right) .
\end{gathered}
$$

In order to write terms in (17) in a compact form, define the matrix function $\bar{H}(x) \in \mathcal{C}^{1}[x], \bar{H}: \mathbb{R}_{\geq 0} \times(a, b) \rightarrow \mathbb{S}^{n_{\alpha}}$ to be the matrix satisfying

$$
\begin{aligned}
& \left(D^{\alpha} u\right)^{\prime} \bar{H}(t, x)\left(D^{\alpha} u\right) \\
& :=\left(D^{\alpha-1} u\right)^{\prime}\left[\frac{\partial H(t, x)}{\partial x}\left(D^{\alpha-1} u\right)+2 H(t, x)\left(D^{\alpha} u\right)\right] .
\end{aligned}
$$

At this stage, we are ready to present conditions to verify inequality (15) for $u$ satisfying (16). Let $T \geq 0$.

Proposition 1: Consider integral inequality (15). If there exist a matrix polynomial $H(t, x)$ and a matrix polynomial $\bar{H}(t, x)$ as defined in (18) such that

$$
F(t, x)+\bar{H}(t, x) \geq 0, \forall t \in[0, T], x \in(a, b),
$$

and

$$
\begin{gathered}
\left(D^{\alpha-1} u(t, b)\right)^{\prime}\left(H(t, b)+F_{1}(t)\right)\left(D^{\alpha-1} u(t, b)\right) \\
-\left(D^{\alpha-1} u(t, a)\right)^{\prime}\left(H(t, a)+F_{0}(t)\right)\left(D^{\alpha-1} u(t, a)\right) \leq 0 \\
\forall u \in \mathcal{U}_{s}(Q)
\end{gathered}
$$

then $\mathcal{F} \geq 0$ for all $u \in \mathcal{U}_{s}(Q)$ and $t \in[0, T]$.

Proof: The proof follows the same lines as the proof of Theorem 1 in [14]. Multiplying left and right sides of (19) by $\left(D^{\alpha-1} u\right)^{\prime}$ and $D^{\alpha-1} u$, respectively, and integrating from $a$ to $b$ yields

$$
\begin{aligned}
& \int_{a}^{b}\left(D^{\alpha} u\right)^{\prime} F(t, x)\left(D^{\alpha} u\right) \mathrm{d} x \\
& \quad+\int_{a}^{b}\left(D^{\alpha} u\right)^{\prime} \bar{H}(t, x)\left(D^{\alpha} u\right) \mathrm{d} x \geq 0, t \in[0, T] .
\end{aligned}
$$

Furthermore, substituting (18) into (17) gives

$$
\begin{aligned}
\int_{a}^{b}\left(D^{\alpha} u\right)^{\prime} & \bar{H}(t, x)\left(D^{\alpha} u\right) \mathrm{d} x= \\
& \left(D^{\alpha-1} u(t, b)\right)^{\prime} H(t, b)\left(D^{\alpha-1} u(t, b)\right) \\
& -\left(D^{\alpha-1} u(t, a)\right)^{\prime} H(t, a)\left(D^{\alpha-1} u(t, a)\right) .
\end{aligned}
$$

Substituting (22) into (21) yields

$$
\begin{aligned}
& \int_{a}^{b}\left(D^{\alpha} u\right)^{\prime} F(t, x)\left(D^{\alpha} u\right) \mathrm{d} x \\
& \quad+\left(D^{\alpha-1} u(t, b)\right)^{\prime} H(t, b)\left(D^{\alpha-1} u(t, b)\right) \\
& -\left(D^{\alpha-1} u(t, a)\right)^{\prime} H(t, a)\left(D^{\alpha-1} u(t, a)\right) \geq 0, t \in[0, T] .
\end{aligned}
$$

Since (20) holds, we have

$$
\begin{aligned}
& \left(D^{\alpha-1} u(t, b)\right)^{\prime} H(t, b)\left(D^{\alpha-1} u(t, b)\right) \\
& \quad-\left(D^{\alpha-1} u(t, a)\right)^{\prime} H(t, a)\left(D^{\alpha-1} u(t, a)\right) \\
& \leq-\left[\left(D^{\alpha-1} u(t, b)\right)^{\prime} F_{1}(t)\left(D^{\alpha-1} u(t, b)\right)\right. \\
& \left.-\left(D^{\alpha-1} u(t, a)\right)^{\prime} F_{0}(t)\left(D^{\alpha-1} u(t, a)\right)\right], \quad \forall u \in \mathcal{U}_{s}(Q) .
\end{aligned}
$$

Then, it follows that $\mathcal{M}$, as in (15), is greater than the left hand side of inequality (23). Since the left hand side of inequality (23) is non-negative, we can conclude that $\mathcal{M}$ is non-negative, as well.

2) Barrier Functionals as Integral Functionals: Note that the method described in the previous subsection requires the problem data to be polynomial. Furthermore, in Theorem 2 and Corollary 1 the only assumption on the barrier functional was $B(t, u(t, x)) \in \mathcal{C}^{1}[t]$, however, in order to provide a computational formulation based on SDPs, we consider the following structure for the barrier functionals

$$
B(t, u(t, x))=\int_{\Omega} b\left(t, x, u, \partial_{x} u, \partial_{x}^{2} u\right) \mathrm{d} x,
$$

with $b \in \mathcal{R}\left[t, x, u, \partial_{x} u, \partial_{x}^{2} u\right]$, that is, $\mathscr{B}(t): u \mapsto$ $\int_{\Omega} b\left(t, x, u, \partial_{x} u, \partial_{x}^{2} u\right) \mathrm{d} x$ as in (7).

Then, in order to solve the associated integral inequalities with SDPs, we assume that $f, g \in \mathcal{R}[s, X]$ as in (1), $c, h \in$ $\mathcal{R}[t, x]$ as in (2) and $q \in \mathcal{R}[x]$ as in (2).

We consider the following undesirable set

$$
\begin{gathered}
\mathcal{Y}_{u}=\left\{u \in \mathcal{U} \mid \int_{\Omega} p_{i}\left(t, x, u, \partial_{x} u, \partial_{x}^{2} u\right) \mathrm{d} x \leq 0,\right. \\
\left.\int_{\Omega} \tilde{p}_{j}\left(t, x, u, \partial_{x} u, \partial_{x}^{2} u\right) \mathrm{d} x=0,(i, j) \in I_{u} \times J_{u}\right\},
\end{gathered}
$$

where $\left\{p_{i}: \mathcal{C}^{1}\left(\mathbb{R}_{\geq 0} \times \Omega \times \mathbb{R}^{3 n} ; \mathbb{R}\right)\right\}_{i \in I_{u}}$ and $\left\{\tilde{p}_{j}\right.$ : $\left.\mathcal{C}^{1}\left(\mathbb{R}_{\geq 0} \times \Omega \times \mathbb{R}^{3 n} ; \mathbb{R}\right)\right\}_{j \in J_{u}}, I_{u}, J_{u} \subset \mathbb{N}_{\geq 0}$ are index sets.

For the set of terminal or initial conditions in the form of (8), we just need to substitute $u(T, x)=q(x)$ in the barrier functional, i.e.,

$$
B(T, u(T, x))=\int_{\Omega} b\left(T, x, q(x), \partial_{x} q(x), \partial_{x}^{2} q(x)\right) \mathrm{d} x .
$$

Under the above assumptions, the conditions of Theorem 2, and Corollary 1 become integral inequalities with 
polynomial integrands, which are required to hold in sets defined by integral inequality constraints. For example, substituting (25) in (9a) yields

$$
\begin{array}{r}
\int_{\Omega} b\left(t_{0}, x, D^{2} u\left(t_{0}, x\right)\right) \mathrm{d} x \\
-\int_{\Omega} b\left(T, x, D^{2} u(T, x)\right) \mathrm{d} x>0, \\
\forall u\left(t_{0}, x\right) \in \mathcal{Y}_{u},
\end{array}
$$

in which $u\left(t_{0}, x\right) \in \mathcal{Y}_{u}$ is a set of integral inequality/equality constraints given by (26). The conventional way of including an integral constraint is to multiply the integral constraint by a constant and add it to the inequality. This method was proposed in [30] to consider constraints such as integral quadratic constraints (IQCs). However, it was observed that the method is too conservative. To address this problem in a less conservative manner, we propose an S-procedure for integral inequalities. The following Theorem is instrumental in solving constrained inequalities similar to (27).

Theorem 3 (S-Procedure for Integral Inequalities): Consider the following integral inequality

$$
\int_{a}^{b} f\left(t, x, D^{\psi_{1}} u\right) \mathrm{d} x \geq 0
$$

subject to

$$
\begin{aligned}
& u \in \mathcal{U}^{\prime}=\left\{u \mid \int_{a}^{b} g_{1}\left(t, x, D^{\psi_{2}} u\right) \mathrm{d} x \leq 0,\right. \\
&\left.\int_{a}^{b} g_{2}\left(t, x, D^{\psi_{2}} u\right) \mathrm{d} x=0\right\},
\end{aligned}
$$

where $f \in \mathcal{R}\left[t, x, D^{\psi_{1}} u\right]$, and $g_{1}, g_{2} \in \mathcal{R}\left[t, x, D^{\psi_{2}} u\right]$. Let

$$
v_{i}(t, x)=\int_{a}^{x} g_{i}\left(t, x, D^{\psi_{2}} u\right) \mathrm{d} x, i=1,2,
$$

satisfying

$$
\left\{\begin{array}{l}
v_{1}(t, a)=0 \\
v_{1}(t, b) \leq 0 \\
v_{2}(t, a)=0 \\
v_{2}(t, b)=0
\end{array}\right.
$$

If there exist $F(t, x) \in \mathcal{R}[t, x]$, and $N(t, x) \in \mathcal{R}[t, x]$ such that

$$
\begin{aligned}
& \int_{a}^{b}\left(f\left(t, x, D^{\psi_{1}} u\right)\right. \\
& \quad+F(t, x)\left(\partial_{x} v_{1}(t, x)-g_{1}\left(t, x, D^{\psi_{2}} u\right)\right) \\
& \left.+N(t, x)\left(\partial_{x} v_{2}(t, x)-g_{2}\left(t, x, D^{\psi_{2}} u\right)\right)\right) \mathrm{d} x \geq 0
\end{aligned}
$$

then (28) holds subject to (29).

Proof: Defining new dependent variables (30) satisfying (31) implies

$$
\int_{a}^{b} g_{1}\left(t, x, D^{\psi_{1}} u\right) \mathrm{d} x \leq 0, \quad \int_{a}^{b} g_{2}\left(t, x, D^{\psi_{1}} u\right) \mathrm{d} x=0,
$$

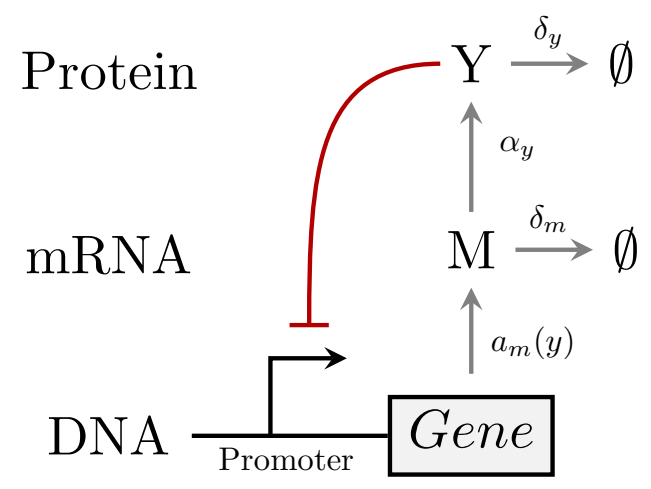

Fig. 1: Diagram of negative autoregulation. The grey arrows represent the chemical reactions (33). The red barred line represents the repressive behaviour of the protein $\mathrm{Y}$ on the transcription of the gene.

which, in turn ensures that (29) holds. In addition, the partial derivative of $v_{1}$ and $v_{2}$ with respect to $x$ is given by

$$
\partial_{x} v_{i}(t, x)-g_{i}\left(t, x, D^{\psi_{2}} u\right)=0, i=1,2,
$$

which is identically zero for all $t, \partial_{x} v_{1}(t, x), \partial_{x} v_{2}(t, x)$, $D^{\psi_{2}} u(t, x) \in \mathbb{R}$ and $x \in(a, b)$. Then, for any $F(t, x), N(t, x) \in \mathcal{R}[t, x]$, we have

$$
\begin{aligned}
& \int_{a}^{b} F(t, x)\left(\partial_{x} v_{1}(t, x)-g_{1}\left(t, x, D^{\psi_{2}} u\right)\right) \mathrm{d} x \\
& +\int_{a}^{b} N(t, x)\left(\partial_{x} v_{2}(t, x)-g_{2}\left(t, x, D^{\psi_{2}} u\right)\right) \mathrm{d} x=0 .
\end{aligned}
$$

Thus, we have

$$
\begin{aligned}
\int_{a}^{b} f(t, & \left.x, D^{\psi_{1}} u\right) \mathrm{d} x=\int_{a}^{b}\left(f\left(t, x, D^{\psi_{1}} u\right)\right. \\
& +F(t, x)\left(\partial_{x} v_{1}(t, x)-g_{1}\left(t, x, D^{\psi_{2}} u\right)\right) \\
& \left.+N(t, x)\left(\partial_{x} v_{2}(t, x)-g_{2}\left(t, x, D^{\psi_{2}} u\right)\right)\right) \mathrm{d} x .
\end{aligned}
$$

Therefore, if inequality (32) is satisfied with dependent variables (30) satisfying (31). It follows that (28) holds subject to (29).

Remark 5: After applying Theorem 3, inequality (32) should be checked via the result in Proposition 1.

\section{Case Study: Negative Autoregulation}

In the following, a model of negative autoregulation is developed using standard principles. Then, using the method developed in Section III, we bound a functional of the second moment.

\section{A. The Model}

This section will begin with construction of a standard two state model of negative autoregulation [31], including both transcription (formation of mRNA from DNA) and translation (formation of protein from mRNA). The other mechanisms included are: 1) the degradation of mRNA, 2) the dilution of protein, and 3) the interaction between protein 
and DNA that accounts for the negative autoregulation. We also demonstrate the standard method of singular perturbation used to reduce this model to one state. The chemical equations describing negative autoregulation are as follows:

$$
\begin{array}{r}
\emptyset \stackrel{\mathrm{a}_{m}(\mathrm{y})}{\longrightarrow} \mathrm{M} \stackrel{\delta_{m}}{\longrightarrow} \emptyset \\
\mathrm{M} \stackrel{\alpha_{\mathrm{y}}}{\longrightarrow} \mathrm{Y}+\mathrm{M} \\
\mathrm{Y} \stackrel{\delta_{\mathrm{y}}}{\longrightarrow} \emptyset
\end{array}
$$

where $\mathrm{M}$ and $\mathrm{Y}$ are the chemical species mRNA and protein respectively, $\alpha_{y}$ is the translation rate, $\delta_{m}$ is the degradation rate of mRNA, $\delta_{y}$ is dilution rate of the protein. The transcription rate $a_{m}(y)$ is dependent on $y$, the concentration of the protein. It is through this term that the feedback occurs. The interaction between gene and protein and its effect on transcription can be modelled using the classic Hill Function $a_{m}(y)=\alpha_{m} /\left(1+K y^{n}\right)$, where $K$ accounts for the affinity between the promoter and transcription factor and $n$ is the Hill Coefficient [32]. This system is presented in Figure 1.

From (33), using standard techniques [31], the following two state ODE model can be constructed:

$$
\begin{aligned}
\frac{\mathrm{d} y}{\mathrm{~d} t} & =\alpha_{y} m-\delta_{y} y \\
\frac{\mathrm{d} m}{\mathrm{~d} t} & =\frac{\alpha_{m}}{1+K y^{n}}-\delta_{m} m
\end{aligned}
$$

where $y$ is the concentration of protein and $m$ is the concentration of mRNA and the parameters are identical to those in (33).

To put this model in the standard single state form used to study the system [18], [19], [33], singular perturbation is used. The following change of variables is performed:

$$
\begin{array}{rr}
\bar{y}=\gamma y & \phi=\frac{\delta_{m}}{\alpha_{m}} \\
\bar{m}=\phi m & \gamma=\frac{\delta_{y} \delta_{m}}{\alpha_{y} \alpha_{m}} \\
\tau=\theta t & K^{\prime}=K \gamma^{-n}
\end{array}
$$

yielding the system

$$
\begin{aligned}
\frac{\mathrm{d} \bar{y}}{\mathrm{~d} \tau} & =\bar{m}-\bar{y} \\
\epsilon \frac{\mathrm{d} \bar{m}}{\mathrm{~d} \tau} & =\frac{1}{1+K^{\prime} \bar{y}^{n}}-\bar{m},
\end{aligned}
$$

where $\epsilon=\delta_{y} / \delta_{m}<1$ is the singular perturbation parameter and is small because the degradation rate of mRNA (on the order of minutes [34]) is assumed to be much higher that the dilution rate of the protein (on the order of an hour [19]). In the limit $\epsilon \rightarrow 0$, the second equation vanishes yielding $\bar{m}=\frac{1}{1+K^{\prime} \bar{y}^{n}}$. This allows for the reduction of the whole system to one equation:

$$
\frac{\mathrm{d} \bar{y}}{\mathrm{~d} \tau}=\frac{1}{1+K^{\prime} \bar{y}^{n}}-\bar{y}
$$

Transforming back to the original variables finally gives us the one state equation for the autorepression system:

$$
\frac{\mathrm{d} y}{\mathrm{~d} t}=\frac{\alpha_{y}^{\prime}}{1+K y^{n}}-\delta_{y} y
$$

TABLE I: Parameters to simulate negative autoregulation.

\begin{tabular}{c|l|c|l} 
Param. & Val. & Param. & Val. \\
\hline$\alpha_{y}$ & $5 \mathrm{~s}^{-1}$ & $\delta_{y}$ & $0.025 \mathrm{~s}^{-1}$ \\
$\alpha_{m}$ & $1 \mathrm{nMs}^{-1}$ & $\delta_{m}$ & $0.25 \mathrm{~s}^{-1}$ \\
$K$ & $200 \mathrm{nM}^{-2}$ & $n$ & 2
\end{tabular}

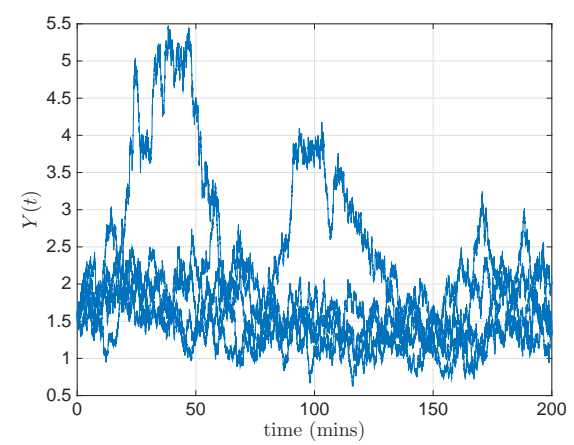

Fig. 2: Five trajectories of $\operatorname{SDE}(40)$ starting at $Y=y^{*}$.

where $\alpha_{y}^{\prime}=\frac{\alpha_{y} \alpha_{m}}{\delta_{m}}$. This yields the explicit relationship between the one state and two state models. The one state system can be represented by the following chemical reactions:

$$
\emptyset \stackrel{\mathrm{a}_{\mathrm{y}}(\mathrm{y})}{\longrightarrow} \mathrm{Y} \stackrel{\delta_{\mathrm{y}}}{\longrightarrow} \emptyset
$$

where $a_{y}(y)=\frac{\alpha_{y}^{\prime}}{1+K y^{n}}$. Typical parameters used to simulate the system are presented in Table I and we use these values in the following. More information on the modelling gene regulatory networks is readily available in [31] and [35]. System (38) has an steady state at $y^{*}=1.5864 \mathrm{nM}$, which can be obtained by solving the following nonlinear equation using MATLAB

$$
\frac{\alpha_{y}^{\prime}}{1+K y^{* n}}-\delta_{y} y^{*}=0
$$

In order to account for the biochemical noise affecting the system, we consider a stochastic version of the one state system (38). We follow the methodology given in [13] and, instead of introducing additive noise, we consider multiplicative noise originating from intrinsic biological fluctuations. The SDE model is described as follows

$$
\begin{array}{r}
\mathrm{d} Y=\left(\frac{\alpha_{y}^{\prime}}{1+K Y^{n}}-\delta_{y} Y\right) \mathrm{d} s+\sqrt{\frac{\alpha_{y}^{\prime}}{1+K Y^{n}}} \mathrm{~d} W_{1} \\
+\sqrt{\delta_{y} Y} \mathrm{~d} W_{2}, s \in[t, T],
\end{array}
$$

where $W_{1}$ and $W_{2}$ are two independent Wiener processes. It can be observed that, although $y^{*}=1.5864 \mathrm{nM}$ is a stationary solution to the deterministic system (38), system (40) is subject to stochastic fluctuations around this stationary solution. This phenomenon tallies with experimental data [18], [19], [20], [21], [33]. Fig. 2 shows five different solutions of SDE (40) starting at $Y=y^{*}$.

\section{B. Bounding the Second Moment}

We are interested in finding bounds on the second moment of $Y$, as it allows us to compare computational estimates of 
the noise with experimental data. To this end, we consider (2) with $c=0, h=\frac{1}{2 T} y^{2}$ and $q=\frac{y^{2}}{2}$. Then the corresponding backward PDE is given by

$$
\begin{aligned}
-\partial_{t} u(t, y)= & \left(\frac{\alpha_{y}^{\prime}}{1+K y^{n}}+\delta_{y} y\right) \partial_{y}^{2} u(t, y) \\
& +\left(\frac{\alpha_{y}^{\prime}}{1+K y^{n}}-\delta_{y} y\right) \partial_{y} u(t, y)+\frac{y^{2}}{2 T} \\
u(T, y)= & \frac{y^{2}}{2}, \quad t \in[0, T], y \in \Omega
\end{aligned}
$$

We assume $\Omega$ is chosen such that for all $s \in[0, T], Y(s) \in \Omega$ (see Remark 3). In this case, based on 5000 Monte Carlo simulations of (40), $\Omega=[0,10 \mathrm{nM}]$ satisfies this requirement for $T=300$ mins. From Theorem 1 , we have

$$
u(t, y)=\frac{1}{2} E\left\{\frac{1}{T} \int_{t}^{T} Y^{2}(s) \mathrm{d} s+Y^{2}(T) \mid Y(t)=y\right\} .
$$

The boundary conditions are set to $u(t, 0)=u(t, 10)=0$. Next, we find bounds on the following functional

$$
u\left(0, y^{*}\right)=\frac{1}{2} E\left\{\frac{1}{T} \int_{0}^{T} Y^{2}(s) \mathrm{d} s+Y^{2}(T) \mid Y(0)=y^{*}\right\},
$$

i.e., the average plus the terminal cost of the second moment around the stationary solution. In order to find bounds on the above functional, we consider the following barrier functional

$$
B(t, u(t, y))=\int_{\Omega}\left(1+K y^{n}\right) b(t, y) u^{2}(t, y) \mathrm{d} y .
$$

Notice with this choice of barrier functional both inequalities (9a) and (9b) are integral inequalities with polynomial integrands. Based on Theorem 2 and solving optimisation problem (14), we obtain the bounds given in Table II, where $\operatorname{deg}(b(t, y))$ is the degree of the certificate $b(t, y)$ in $t$ and $y$. For degree 8 , we found a bound of $u\left(0, y^{*}\right) \leq 3.3791$ for which, using (44) and ignoring the terms smaller than $10^{-4}$, the following certificate $b(t, y)$ was constructed

$$
\begin{aligned}
b(t, y)= & -22.64 y^{8}+279.7 t y^{7}+453.3 y^{7}-393.6 t^{2} y^{6}+189.6 t y^{6} \\
& -463.2 y^{6}-27.73 t^{3} y^{5}-20.47 t^{2} y^{5}+27.19 t y^{5}-4.018 y^{5} \\
& -308.7 t^{4} y^{4}+13.5 t^{3} y^{4}-185.9 t^{2} y^{4}+102.9 t y^{4}-418.9 y^{4} \\
& -18.67 t^{5} y^{3}-19.0 t^{4} y^{3}-14.63 t^{3} y^{3}-17.96 t^{2} y^{3}-6.14 t y^{3} \\
& -38.55 y^{3}-356.5 t^{6} y^{2}+6.329 t^{5} y^{2}-166.7 t^{4} y^{2}+44.79 t^{3} y^{2} \\
& -120.8 t^{2} y^{2}+194.6 t y^{2}-436.7 y^{2}-5.959 t^{7} y-5.85 t^{6} y \\
& -5.566 t^{5} y-6.046 t^{4} y-4.284 t^{3} y-4.482 t^{2} y+0.05402 t y \\
& -6.418 y-966.3 t^{8}+22.7 t^{7}-404.3 t^{6}+101.9 t^{5} \\
& -177.3 t^{4}+293.2 t^{3}-57.82 t^{2}+1026.0 t-1137 .
\end{aligned}
$$

This is consistent with the result obtained from 5000 Monte Carlo simulations as illustrated in Fig. 3, where the value for $u\left(0, y^{*}\right)$ from Monte Carlo simulations is 3.3417 .

Employing the bound on the second moment of $3.3791 \mathrm{nM}^{2}$ and the mean steady state value of the system of $y^{*}=1.5864 \mathrm{nM}$, the coefficient of variance was calculated to be $v_{c}=0.5854$. Looking at [21], page 5, Fig. 4D 'TG-nf',
TABLE II: Obtained bounds on functional (43).

\begin{tabular}{c|c|c|c|c}
$\operatorname{deg}(b(t, y))$ & 2 & 4 & 6 & 8 \\
\hline bound $\left(\mathrm{nM}^{2}\right)$ & 8.7401 & 6.3642 & 4.4562 & 3.3791
\end{tabular}

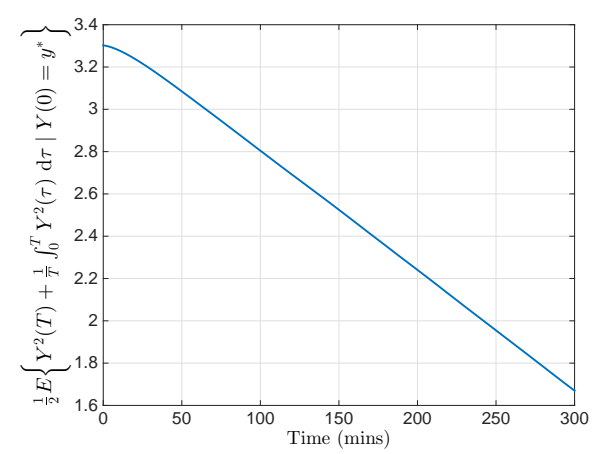

Fig. 3: The evolution of the cost functional (42) with $T=$ 300 obtained from 5000 Monte Carlo simulations.

which shows coefficient of variance data from experiments on a system equivalent to the one studied here, the values are in close agreement.

\section{CONCLUSIONS}

We proposed a method for bounding state functionals of a class of nonlinear stochastic systems based on barrier functionals. The method can be cast as solving SDPs in the case of polynomial data. The method was applied to a stochastic model of negative autoregulation. It was shown that the resulting bound on the second moment yielded a coefficient of variance that closely mirrored experimental data. The Gillespie algorithm [1], which is commonly used to model such processes can provide estimates of moments, but these are never bounded with certificates. Further application of such techniques to more complete and complex models of biological systems of more than one state would be a powerful tool in the study of these systems. It would allow for more accurate prediction of stochastic behaviour and yield further insight into the relative roles of the various modelling techniques employed in fields such as systems and synthetic biology.

\section{REFERENCES}

[1] D. T. Gillespie, "A general method for numerically simulating the stochastic time evolution of coupled chemical reactions," Journal of Computational Physics, no. 4, pp. 403-434, 1976.

[2] K. Burrage, P. Burrage, and T. Mitsui, "Numerical solutions of stochastic differential equations: implementation and stability issues," Journal of Computational and Applied Mathematics, no. 1\&2, pp. 171-182, 2000.

[3] R. Khasminskii, Stochastic Stability of Differential Equations, ser. Stoch. Model. Appl. Probab. New York, US: Springer, 2012, no. 66.

[4] X. Mao, Stochastic Differential Equations and Applications, 2nd ed. Cambridge, UK: Woodhead Publishing, 2012.

[5] R. C. Merton, "Theory of rational option pricing," The Bell Journal of Economics and Management Science, no. 1, pp. 141-183, 1973.

[6] M. Soltani, C. Vargas-Garcia, and A. Singh, "Conditional moment closure schemes for studying stochastic dynamics of genetic circuits," Biomedical Circuits and Systems, IEEE Transactions on, no. 4, pp. 518-526, Aug 2015. 
[7] I. Krishnarajah, A. Cook, G. Marion, and G. Gibson, "Novel moment closure approximations in stochastic epidemics," Bulletin of Mathematical Biology, no. 4, pp. 855-873, 2005.

[8] C. Gillespie, "Moment-closure approximations for mass-action models," Systems Biology, IET, no. 1, pp. 52-58, January 2009.

[9] A. Singh and J. Hespanha, "Approximate moment dynamics for chemically reacting systems," Automatic Control, IEEE Transactions on, no. 2, pp. 414-418, Feb 2011.

[10] M. Ahmadi, G. Valmorbida, and A. Papachristodoulou, "Barrier functionals for output functional estimation of PDEs," in American Control Conference (ACC), 2015, July 2015, pp. 2594-2599.

[11] S. Prajna, "Barrier certificates for nonlinear model validation," Automatica, no. 1, pp. 117 - 126, 2006.

[12] S. Prajna, A. Jadbabaie, and G. J. Pappas, "A framework for worst-case and stochastic safety verification using barrier certificates," Automatic Control, IEEE Transactions on, no. 8, pp. 1415-1428, Aug 2007.

[13] H. El-Samad, S. Prajna, A. Papachristodoulou, J. Doyle, and M. Khammash, "Advanced methods and algorithms for biological networks analysis," Proceedings of the IEEE, no. 4, pp. 832-853, April 2006.

[14] G. Valmorbida, M. Ahmadi, and A. Papachristodoulou, "Stability analysis for a class of partial differential equations via semi-definite programming," IEEE Transactions on Automatic Control, 2016, to Appear, DOI:10.1109/TAC.2015.2479135.

[15] M. Ahmadi, G. Valmorbida, and A. Papachristodoulou, "Dissipation inequalities for the analysis of a class of PDEs," Automatica, pp. 163 - 171, 2016.

[16] T. Afroz and C. L. Beisel, "Understanding and exploiting feedback in synthetic biology," Chemical Engineering Science, pp. 1-12, nov 2013.

[17] A. W. K. Harris, J. A. Dolan, C. L. Kelly, J. Anderson, and A. Papachristodoulou, "Designing Genetic Feedback Controllers," IEEE Transactions on Biomedical Circuits and Systems, no. 4, pp. 475-484, 2015.

[18] A. Becskei and L. Serrano, "Engineering stability in gene networks by autoregulation." Nature, no. June, pp. 590-593, 2000.

[19] N. Rosenfeld, M. B. Elowitz, and U. Alon, "Negative autoregulation speeds the response times of transcription networks," Journal of Molecular Biology, no. 5, pp. 1-7, 2002.

[20] D. W. Austin, M. S. Allen, J. M. McCollum, R. D. Dar, J. R. Wilgus, G. S. Sayler, N. F. Samatova, C. D. Cox, and M. L. Simpson, "Gene network shaping of inherent noise spectra." Nature, no. 7076, pp. 608-11, feb 2006.

[21] Y. Dublanche, K. Michalodimitrakis, N. Kummerer, M. Foglierini, and L. Serrano, "Noise in transcription negative feedback loops: simulation and experimental analysis," Mol.Syst.Biol., no. 1744-4292 (Electronic), p. 41, 2006.

[22] J. Yong and X. Y. Zhou, Stochastic Controls: Hamiltonian Systems and HJB Equations, ser. Applications of Mathematics. Springer, 1999, no. 43.

[23] I. Karatzas and S. E. Shreve, Brownian Motion and Stochastic Calculus, 2nd ed., ser. Graduate Texts in Mathematics. Springer, 1991.

[24] H. Risken, The Fokker-Planck equation: methods of solution and applications. 2nd, Springer-Verlag, 1989.

[25] A. Milias-Argeitis and M. Khammash, "Optimization-based Lyapunov function construction for continuous-time Markov chains with affine transition rates," in Decision and Control (CDC), 2014 IEEE 53rd Annual Conference on, Dec 2014, pp. 4617-4622.

[26] S. P. Meyn and R. L. Tweedie, "Stability of Markovian Processes III: Foster-Lyapunov Criteria for Continuous-Time Processes," Advances in Applied Probability, no. 3, 1993.

[27] N. V. Kampen, Stochastic Processes in Physics and Chemistry, third edition ed. Amsterdam: Elsevier, 2007.

[28] D. Gillespie, "The chemical Langevin equation," Journal of Chemical Physics, no. 1, pp. 297-306, 2000.

[29] G. Valmorbida and A. Papachristodoulou, "Introducing INTSOSTOOLS: A SOSTOOLS plug-in for integral inequalities," in Control Conference (ECC), 2015 European, 2015, pp. 1231-1236.

[30] A. Papachristodoulou and S. Prajna, "On the construction of Lyapunov functions using the sum of squares decomposition," in Decision and Control, 2002, Proceedings of the 41st IEEE Conference on, vol. 3, Dec 2002, pp. 3482-3487.

[31] U. Alon, An introduction to systems biology: design principles of biological circuits. Boca Raton, Florida: Chapman \& Hall, 2006.

[32] A. Hill, "The possible effects of the aggregation of the molecules of haemoglobin on its dissociation curves," J Physiol (Lond), 1910.
[33] D. Nevozhay, R. M. Adams, K. F. Murphy, K. Josic, and G. Balázsi, "Negative autoregulation linearizes the dose-response and suppresses the heterogeneity of gene expression." Proceedings of the National Academy of Sciences of the United States of America, no. 13, pp. 5123-8, 2009

[34] L. L. Granger, E. B. O’Hara, R. F. Wang, F. V. Meffen, K. Armstrong, S. D. Yancey, P. Babitzke, and S. R. Kushner, "The Escherichia coli mrsC gene is required for cell growth and mRNA decay." Journal of bacteriology, no. 7, pp. 1920-1928, 1998.

[35] B. Ingalls, Mathematical Modelling in Systems Biology: An Introduction. Cambridge, Massachusetts: The MIT Press, 2013. 